\title{
Asymptotic Behavior and Stability of Stochastic SIR Model with Variable Diffusion Rates
}

\author{
Xianhua Xie', Li Ma1,2, Jingfei Xu ${ }^{1 *}$ \\ ${ }^{1}$ Key Laboratory of Jiangxi Province for Numerical Simulation and Emulation Techniques, Gannan Normal University, Ganzhou, \\ China \\ ${ }^{2}$ College of Mathematics and Econometrics, Hunan University, Changsha, China \\ Email:xxianhua@sina.com.cn,mali1983@hnu.edu.cn, *xujingfei2006@163.com.cn
}

How to cite this paper: Xie, X.H., Ma, L. and Xu, J.F. (2017) Asymptotic Behavior and Stability of Stochastic SIR Model with Variable Diffusion Rates. Applied Mathematics, 8, 1031-1044. https://doi.org/10.4236/am.2017.88080

Received: May 12, 2017

Accepted: August 4, 2017

Published: August 7, 2017

Copyright $\odot 2017$ by authors and Scientific Research Publishing Inc. This work is licensed under the Creative Commons Attribution International License (CC BY 4.0).

http://creativecommons.org/licenses/by/4.0/

\begin{abstract}
In this paper, we propose random fluctuation on contact and recovery rates in deterministic SIR model with disease deaths in nonparametric manner and derive a new stochastic SIR model with distributed time delay and general diffusion coefficients. By analysis of the introduced model, we obtain the sufficient conditions for the regularity, existence and uniqueness of a global solution by means of Lyapunov function. Moreover, we also investigate the stochastic asymptotic stability of disease free equilibria and endemic equilibria of this model. Finally, we illustrate our general results by applications.
\end{abstract}

\section{Keywords}

SIR Model, Regularity, Lyapunov Function, Stochastic Asymptotic Stability

\section{Introduction}

SIR models are the foundation for a large number of compartmental models in mathematical epidemiology which classify the population into three classes: Susceptible, Infected and Removed (see [1]-[19]). Generally, these models admit two types of equilibrium: disease free and endemic equilibrium. If the disease free equilibrium is asymptotically stable, it implies the disease dies out. If the endemic equilibrium is asymptotically stable, it implies the disease persists in the population at the equilibrium level.

In 1976, Hethcote [13] considered the following deterministic SIR model with disease deaths:

${ }^{*}$ Corresponding author. 


$$
\left\{\begin{array}{l}
S^{\prime}(t)=-\beta S(t) I(t)+\mu(K-S(t)) \\
I^{\prime}(t)=\beta S(t) I(t)-(\mu+\alpha) I(t) \\
R^{\prime}(t)=\alpha I(t)-\mu R(t)
\end{array}\right.
$$

In Equation (1), $S(t), I(t), R(t)$ denote the number of the individuals susceptible to the disease, of infected members and of members who have been removed from the population, respectively. The model (1) is based on the following assumptions:

i) The population considered has a constant size $K$, that is, $S(t)+I(t)+R(t)=K$ for all $t$;

ii) Births and deaths occur at equal rates $\mu$ in $K$. All the newborns are susceptible. $\mu$ is called a daily death removal rate;

iii) $\quad \beta$ is the daily contact rate, i.e., the average number of contacts per infective per day. A contact of an infective is an interaction which results in infection of the other individual if it is susceptible;

iv) $\alpha$ is the daily recovery removal rate of the infective. Of course, $\beta, \mu, \alpha \in R^{+}$.

In [8], Beretta and Takeuchi pointed out that when a susceptible vector is infected by a person, there is a time $\tau>0$ during which the infectious agents develop in the vector and it is only after that time that the infected vector becomes itself infectious, and proposed the following model

$$
\left\{\begin{array}{l}
S^{\prime}(t)=-\beta S(t) \int_{0}^{h} f(s) I(t-s) \mathrm{d} s+\mu(K-S(t)) \\
I^{\prime}(t)=\beta S(t) \int_{0}^{h} f(s) I(t-s) \mathrm{d} s-(\mu+\alpha+\gamma) I(t), \\
R^{\prime}(t)=\alpha I(t)-\mu R(t)
\end{array}\right.
$$

where $f(s)$ is a non-negative function which is square integrable on $[0, h]$ and satisfies

$$
\int_{0}^{h} f(s) \mathrm{d} s=1, \quad \int_{0}^{h} s f(s) \mathrm{d} s<+\infty,
$$

and the non-negative constant $h$ is the time delay, $\beta S(t) \int_{0}^{h} f(s) I(t-s) \mathrm{d} s$ can be viewed as the force of infection at time $t$.

In fact, all infectious diseases are subject to randomness in terms of the nature of transmission. Recently, Tornatore et al. [16] investigate the dynamics of system (2) by perturbing the functional contact rates and modified (2) as:

$$
\left\{\begin{array}{l}
\mathrm{d} S(t)=\left[-\beta S(t) \int_{0}^{h} f(s) I(t-s) \mathrm{d} s-\mu S(t)+\mu\right] \mathrm{d} t-\sigma S(t) \int_{0}^{h} f(s) I(t-s) \mathrm{d} s \mathrm{~d} W(t) \\
\mathrm{d} I(t)=\left[\beta S(t) \int_{0}^{h} f(s) I(t-s) \mathrm{d} s-(\mu+\alpha) I(t)\right]+\sigma S(t) \int_{0}^{h} f(s) I(t-s) \mathrm{d} s \mathrm{~d} W(t) \\
\mathrm{d} R(t)=\alpha I(t)-\mu R(t),
\end{array}\right.
$$

where $\sigma$ is a positive constant and $W$ is a real Wiener process defined on a stochastic basis $\left(\Omega, \mathfrak{F},\left(\mathfrak{F}_{t}\right)_{t \geq 0}, P\right)$. They only proved the stability of disease-free equilibrium under some given condition. Along these clues, we propose a 
stochastic SIR model with deaths and varying contact and recovery rates, where the introduced model covers general diffusion coefficients (functional contact and recovery rates).

In order to make the SIR system (2) more realistic, we consider the case of $S(t)+I(t)+R(t) \leq K$ and we perturbed the deterministic system (2) by a white noise and obtained a stochastic counterpart by replacing the rates $\beta$ by $\beta+F_{1}(S(t), I(t), R(t)) \frac{\mathrm{d} W_{1}(t)}{\mathrm{d} t}$ and $\alpha \quad$ by $\alpha+F_{2}(S(t), I(t), R(t)) \frac{\mathrm{d} W_{2}(t)}{\mathrm{d} t}$, and hence we modify the SIR system (2) as the following model:

$$
\left\{\begin{aligned}
\mathrm{d} S(t)= & {\left[-\beta S(t) \int_{0}^{h} f(s) I(t-s) \mathrm{d} s+\mu(K-S(t))\right] \mathrm{d} t-S(t) \int_{0}^{h} f(s) I(t-s) \mathrm{d} s } \\
& \times F_{1}(S(t), I(t), R(t)) \mathrm{d} W_{1}(t), \\
\mathrm{d} I(t)= & {\left[\beta S(t) \int_{0}^{h} f(s) I(t-s) \mathrm{d} s-(\mu+\gamma+\alpha) I(t)\right]+S(t) \int_{0}^{h} f(s) I(t-s) \mathrm{d} s } \\
& \times F_{1}(S(t), I(t), R(t)) \mathrm{d} W_{1}(t)-\int_{0}^{h} f(s) I(t-s) \mathrm{d} s F_{2}(S(t), I(t), R(t)) \mathrm{d} W_{2}(t) \\
\mathrm{d} R(t)= & \alpha I(t)-\mu R(t)+\int_{0}^{h} f(s) I(t-s) \mathrm{d} s F_{2}(S(t), I(t), R(t)) d W_{2}(t),
\end{aligned}\right.
$$

where $\alpha, \beta, \mu, K$ have the same meaning as model $(2), W_{i}(t)(i=1,2)$ are real Wiener processes and i.i.d which defined on a filtered complete probability space $\left(\Omega, \mathfrak{F},\left(\mathfrak{F}_{t}\right)_{t \geq 0}, P\right)$. Here, we introduce other two new general stochastic terms: functions $F_{i}(i=1,2)$ which are locally Lipschitz continuous defined on

$$
\mathbb{D}=\left\{(S, I, R) \in \mathbb{R}^{3}: S \geq 0, I \geq 0, R \geq 0, S+I+R \leq K\right\} .
$$

Besides, there are deaths due to disease the total population size may vary in time so that we always assume the total population size is less than $K$ in the context, where $K$ represents a carrying capacity. Note that if we consider the population size is a constant $K$ and the disease-related death rate $\gamma=0$, besides we also take $F_{1}(t)=F_{2}(t)=\sigma$ (where $\sigma$ is a positive constant), the system (3) becomes the model which has been discussed in [16]. In [16], Tornatore proved the stability of disease-free equilibrium under some restricted conditions. However, they didn't consider the dynamics of the endemic equilibrium. It is of great importance from a theoretical point of view to investigate the stability of the endemic equilibrium.

In this paper, we mainly study the stochastic SIR model (5) with distributed delay which has more general diffusion coefficients than model (4)'s. By means of averaged Itô formula and Lyapunov function, we obtain the sufficient conditions for the regularity, existence and uniqueness of a global solution. Furthermore, we also investigate the stochastic asymptotic stability of disease free equilibria and the dynamics of endemic equilibria which has not been discussed in [16].

The remaining parts of the paper are organized as follows: In Section 2, we will give some basic concepts and conclusions. In Section 3, we employ the averaged Itô formula to obtain the regularity, existence and uniqueness of the global solution of SIR model (5). In Section 4, we derive the sufficient condition 
to ensure the global stochastic asymptotic stability of disease free equilibrium in SIR model (5), besides we also consider the stochastic asymptotic stability of endemic equilibrium in Section 5. Finally, we illustrate our general results by applications.

\section{Some Preliminary Definition and Lemmas}

At first, we recall the notation of regularity of continuous time stochastic processes as introduced in [10]. Let $\mathbb{D} \subset \mathbb{R}^{d}(d \geq 1)$ be a fixed closed domain. For Simplicity, we only consider deterministic domains $\mathbb{D} \subset \mathbb{R}^{3}$ in this exposition.

Definition 1. A continuous time stochastic process $\{X(t), t \geq 0\}$ is called regular on $\mathbb{D}$ (or invariant with respect to $\mathbb{D}$ ) if

$$
\forall t \geq 0: \quad P(X(t) \in \mathbb{D})=1
$$

otherwise irregular with respect to $\mathbb{D}$ (or not invariant with respect to $\mathbb{D}$ ).

Consider the $d$-dimensional stochastic differential equation of the form

$$
\mathrm{d} X(t)=f(X(t), t) \mathrm{d} t+g(X(t), t) d W(t),
$$

with an initial value $X\left(t_{0}\right)=X_{0}, t_{0} \leq t \leq T<\infty$ where $f: \mathbb{R}^{d} \times\left[t_{0}, T\right] \rightarrow \mathbb{R}^{d}$ and $f: \mathbb{R}^{d} \times\left[t_{0}, T\right] \rightarrow \mathbb{R}^{d \times m}(m \geq 1)$ are Borel measurable, $W=\{W(t)\}_{t \geq t_{0}}$ is an $\mathbb{R}^{m}$-valued random variable.

Definition 2. The infinitesimal generator $\mathcal{L}$ associated with the $S D E(6)$ is given by

$$
\mathcal{L}=\frac{\partial}{\partial t}+\sum_{i=1}^{d} f_{i}(x, t) \frac{\partial}{\partial x_{i}}+\frac{1}{2} \sum_{i, j=1}^{m}\left(g(x, t) g^{\mathrm{T}}(x, t)\right)_{i j} \frac{\partial^{2}}{\partial x_{i} \partial x_{j}} .
$$

Lemma 2.1. (Regularity Theorem [10]) Let $\mathbb{D}$ and $\mathbb{D}_{n}$ be open sets in $\mathbb{R}^{d}$ with

$$
\mathbb{D}_{n} \subseteq \mathbb{D}_{n+1}, \quad \mathbb{D}_{n} \subseteq \mathbb{D}, \quad \text { and } \quad \mathbb{D}=\bigcup_{n} \mathbb{D}_{n}
$$

and suppose $f$ and $g$ satisfy the existence and uniqueness conditions for solutions of (6) on each set $\left\{(t, x): t>t_{0}, x \in \mathbb{D}_{n}\right\}$. Suppose there is a nonnegative continuous function $V: \mathbb{D} \times\left[t_{0}, T\right] \rightarrow \mathbb{R}^{+}$with continuous partial derivatives and satisfying $\mathcal{L} V \leq c V$ for some positive constant $c$ and $t>t_{0}, x \in \mathbb{D}$. Moreover, if

$$
\inf _{t>t_{0}, x \in \mathbb{D} \mathbb{D}_{n}} V(x, t) \rightarrow \infty \quad \text { as } n \rightarrow \infty,
$$

then $P\left(X_{0} \in \mathbb{D}\right)=1$ for any $X_{0}$ independent of $\sigma(W)$, that is to say the stochastic process $\{X(t), t \geq 0\}$ is called regular on $\mathbb{D}$. Regularity on $\mathbb{D}$ implies boundedness, uniqueness, continuity and Markov property of the strong solution process $X$ of SDE (6) with $X(0)=X_{0}$, and $X(t) \in \mathbb{D}$ for all $t>0$ (a.s.).

Definition 3. The equilibrium solution $x^{*}$ of the SDE (6) is stochastically stable (stable in probability) if for every $\epsilon>0$ and $s \geq t_{0}$ 


$$
\lim _{X_{0} \rightarrow x^{*}} P\left(\sup _{t_{0} \leq s<\infty}\left\|X_{s, X_{0}}(t)\right\| \geq \epsilon\right)=0,
$$

where $X_{s, X_{0}}(t)$ denotes the solution of (6) satisfying $X(s)=X_{0}$ at time $t \geq s$.

Definition 4. The equilibrium solution $x^{*}$ of the SDE (6) is stochastically asymptotically stable (stable in probability) if it is stochastically stable and

$$
\lim _{X_{0} \rightarrow x^{*}} P\left(\lim _{t \rightarrow \infty} X_{s, X_{0}}(t)=x^{*}\right)=1 .
$$

Definition 5. The equilibrium solution $x^{*}$ of the $S D E(6)$ is said to be globally stochastically asymptotically stable (stable in probability) if it is stochastically stable and for every $X_{0}$ and every $s$

$$
P\left(\lim _{t \rightarrow \infty} X_{s, X_{0}}(t)=x^{*}\right)=1 .
$$

Lemma 2.2. ([4]) Assume that $f$ and $g$ satisfy locally Lipschitz-continuous and satisfy linear growth condition and they have continuous coefficients with respect to $t$.

1) Suppose that there exists a positive definite function $V \in C^{2,1}\left(U_{h} \times\left[t_{0}, \infty\right)\right)$, where $U_{h}=\left\{x \in \mathbb{R}^{d}:\left\|x-x^{*}\right\|<h\right\}$ for $h>0$, such that

$$
\mathcal{L} V(x, t) \leq 0, \quad \forall t \geq t_{0}, \quad x \in U_{h} .
$$

Then the equilibrium solution $x^{*}$ of (6) is stochastically stable.

2) In addition, if $V$ is descresent (that is to say there exists a positive definite function $V_{1}$ such that $V(x, t) \leq V_{1}(x)$ for all $\left.x \in U_{h}\right)$ and $\mathcal{L} V(x, t)$ is negative definite, then the equilibrium solution $x^{*}$ is stochastically asymptotically stable.

3) If the assumptions of part (2) hold for a radially unbounded function $V \in C^{2,1}\left(\mathbb{R}^{d} \times\left[t_{0}, \infty\right)\right)$ defined everywhere then the equilibrium solution $x^{*}$ is globally stochastically asymptotically stable.

\section{Existence, Uniqueness and Regularity of Stochastic SIR Model Solution}

Theorem 3.1. Let $\left(S\left(t_{0}\right), I\left(t_{0}\right), R\left(t_{0}\right)\right)=\left(S_{0}, I_{0}, R_{0}\right) \in D=$ $\left\{(S, I, R) \in \mathbb{R}^{3}, t \geq 0: S \geq 0, I \geq 0, R \geq 0, S+I+R \leq K\right\}$, and $\left(S_{0}, I_{0}, R_{0}\right)$ be independent of $\sigma$-algebra $\sigma(W(t), t \geq 0)$. Then, under the condition $(A)$ or $(B)$

a) $\int_{0}^{h} f(s) I(t-s) \mathrm{d} s \leq L I(t)(L>0)$;

b) $\int_{0}^{h} f(s) I(t-s) \mathrm{d} s \leq I(t)+R(t)$;

the stochastic process $\left\{(S(t), I(t), R(t)), t \geq t_{0}\right\}$ governed by Equation (5) is regular on $\mathbb{D}$; i.e. we have $P((S(t), I(t), R(t)) \in \mathbb{D})=1$ for all $t \geq 0$. Moreover, regularity on $\mathbb{D}$ implies stochastic SIR model (5) admits a a unique, continuous-time, Markovian global solution process $\left\{(S(t), I(t), R(t)), t \geq t_{0}\right\}$.

Proof. First we consider the result under the condition of (A). Denote drift term 


$$
b(S, I, R)=\left(\begin{array}{c}
-\beta S \int_{0}^{h} f(s) T(t-s) \mathrm{d} s+\mu(K-S) \\
\beta S \int_{0}^{h} f(s) I(t-s) \mathrm{d} s-(\alpha+\mu+\gamma) I \\
\alpha I-\mu R
\end{array}\right)
$$

and the diffusion term

$$
B(S, I, R)=\left(\begin{array}{ccc}
-S F_{1} \int_{0}^{h} f(s) I(t-s) \mathrm{d} s & 0 & 0 \\
S \int_{0}^{h} f(s) I(t-s) \mathrm{d} s & -I F_{2} & 0 \\
0 & I F_{2} & 0
\end{array}\right) .
$$

Let open domains

$$
\mathbb{D}_{n}:=\left\{(S, I, R): \mathrm{e}^{-n}<S<K-\mathrm{e}^{-n}, \mathrm{e}^{-n}<I<K-\mathrm{e}^{-n}, \mathrm{e}^{-n}<R<K-\mathrm{e}^{-n}, S+I+R \leq K, n \in \mathbb{N}\right\} .
$$

Since Equation (5) is well-defined on $\mathbb{D}$ and $\mathbb{D}_{n}$, and the coefficients $b(S, I, R), B(S, I, R)$ are locally Lipschitz-continuous and satisfy linear growth condition on $\mathbb{D}$, then there exists a unique, bounded and Markovian solution up to random time $\tau(\mathbb{D})$ (or $\tau\left(\mathbb{D}_{n}\right)$ ), where $\tau(\mathbb{D})$ (or $\tau\left(\mathbb{D}_{n}\right.$ ) represents the random time of the first exit of stochastic process $(S(t), I(t), R(t))$ from the domain $\mathbb{D} \quad\left(\right.$ or $\left.\mathbb{D}_{n}\right)$, started in $(S(t), I(t), R(t))=(S(s), I(s), R(s))=$ $\left(S_{0}, I_{0}, R_{0}\right) \in \mathbb{D} \quad\left(\right.$ or $\left.\quad\left(S_{0}, I_{0}, R_{0}\right) \in \mathbb{D}_{n}\right)$ at the initial time $s \in\left[t_{0}, \infty\right)$. To ensure the solution regular, we only prove that $P(\tau(\mathbb{D})=\infty)=1$. a.s. Now, we use function $V \in C^{2}(\mathbb{D})$ defined on $\mathbb{D}$ via

$$
V(S, I, R)=S-\ln S+I-\ln I+(K-S)-\ln (K-S)+(K-R)-\ln (K-R),
$$

and assume that $E V(S, I, R)<\infty$. For $(S, I, R) \in \mathbb{D}$, we have $V(S, I, R) \geq 4$ and for $(S, I, R) \in \mathbb{D} \backslash \mathbb{D}_{n}$, we have

$$
\inf _{(S, I, R) \in \mathbb{D} \mathbb{\mathbb { W } _ { n }}} V(S, I, R)>2 n+2, \quad \text { for } n \in \mathbb{N} .
$$

Define $\mathcal{L}$ as infinitesimal generator as in Definition 2, then calculate

$$
\begin{aligned}
\mathcal{L} V(S, I, R)= & \left.-\beta S \int_{0}^{h} f(s) I(t-s) \mathrm{d} s+\mu(K-S)\right) \frac{\partial V}{\partial S} \\
& +\left(\beta S \int_{0}^{h} f(s) I(t-s) \mathrm{d} s+(\alpha I-\mu R) \frac{\partial V}{\partial R}\right. \\
+ & \frac{1}{2} S^{2}\left(\int_{0}^{h} f(s) I(t-s) \mathrm{d} s\right)^{2} F_{1}^{2}(S, I, R)\left(\frac{\partial^{2} V}{\partial S^{2}}-2 \frac{\partial^{2} V}{\partial S \partial I}+\frac{\partial^{2} V}{\partial I^{2}}\right) \\
& \left.+\frac{1}{2} I^{2} F_{2}^{2}(S, I, R)\left(\frac{\partial^{2} V}{\partial I^{2}}-2 \frac{\partial^{2} V}{\partial I \partial R}+\frac{\partial^{2} V}{\partial R^{2}}\right)-(\alpha+\gamma+\mu) I\right) \frac{\partial V}{\partial I} \\
= & -\beta S \int_{0}^{h} f(s) I(t-s) \mathrm{d} s+\mu(K-S)\left(\frac{1}{K-S}-\frac{1}{S}\right) \\
& +\beta S \int_{0}^{h} f(s) I(t-s) \mathrm{d} s\left(1-\frac{1}{I}\right)+(\alpha I-\mu R)\left(\frac{1}{K-R}-1\right) \\
& +\frac{1}{2} S^{2}\left(\int_{0}^{h} f(s) I(t-s) \mathrm{d} s\right)^{2} F_{1}^{2}(S, I, R)\left(\frac{1}{(K-S)^{2}}+\frac{1}{S^{2}}+\frac{1}{I^{2}}\right)
\end{aligned}
$$




$$
\begin{aligned}
& +\frac{1}{2} I^{2} F_{2}^{2}(S, I, R)\left(\frac{1}{I^{2}}+\frac{1}{(K-R)^{2}}\right)-(\alpha+\gamma+\mu) I\left(1-\frac{1}{I}\right) \\
= & \frac{-\beta S \int_{0}^{h} f(s) I(t-s) \mathrm{d} s}{K-S}+\mu+\beta \int_{0}^{h} f(s) I(t-s) \mathrm{d} s-\frac{\mu(K-S)}{S} \\
& +\beta S \int_{0}^{h} f(s) I(t-s) \mathrm{d} s-\frac{1}{I} \beta S \int_{0}^{h} f(s) I(t-s) \mathrm{d} s+\alpha+\gamma+\mu \\
& +\frac{\alpha I}{K-R}-\alpha I-\frac{\mu R}{K-R}+\mu R+\frac{1}{2}\left(\int_{0}^{h} f(s) I(t-s) \mathrm{d} s\right)^{2} F_{1}^{2}(S, I, R) \\
& +\frac{1}{2} S^{2} \frac{\left(\int_{0}^{h} f(s) I(t-s) \mathrm{d} s\right)^{2} F_{1}^{2}(S, I, R)}{I^{2}} \\
& +\frac{1}{2} \frac{\left(\int_{0}^{h} f(s) I(t-s) \mathrm{d} s\right)^{2} F_{2}^{2}(S, I, R)}{(K-R)^{2}}-(\alpha+\gamma+\mu) I \\
& +\frac{1}{2} S^{2} \frac{\left(\int_{0}^{h} f(s) I(t-s) \mathrm{d} s\right)^{2} F_{1}^{2}(S, I, R)}{(K-S)^{2}} \\
& +\frac{1}{2} \frac{\left(\int_{0}^{h} f(s) I(t-s) \mathrm{d} s\right)^{2} F_{2}^{2}(S, I, R)}{I^{2}} .
\end{aligned}
$$

In view of the condition (A) that $\int_{0}^{h} f(s) I(t-s) \mathrm{d} s \leq L I(t)$, and hence we have

$$
\begin{aligned}
\mathcal{L} V(S, I, R) \leq & 3 \mu+\beta \int_{0}^{h} f(s) I(t-s) \mathrm{d} s+\beta S \int_{0}^{h} f(s) I(t-s) \mathrm{d} s+2 \alpha \\
& +\gamma+\mu R+\sup _{(S, I, R) \in \mathbb{D}}\left\{\frac{3}{2} L K^{2} F_{1}^{2}(S, I, R)+L F_{2}^{2}(S, I, R)\right\} \\
\leq & 3 \mu+\beta L I+\beta S I+2 \alpha+\gamma+\mu R \\
& +\sup _{(S, I, R) \in \mathbb{D}}\left\{\frac{3}{2} L K^{2} F_{1}^{2}(S, I, R)+L F_{2}^{2}(S, I, R)\right\} .
\end{aligned}
$$

If we take

$$
0<c \leq \frac{1}{4}\left[3 \mu+\beta L I+\beta S I+2 \alpha+\gamma+\mu R+\sup _{(S, I, R) \in \mathbb{D}}\left\{\frac{3}{2} L K^{2} F_{1}^{2}(S, I, R)+L F_{2}^{2}(S, I, R)\right\}\right],
$$

therefore $\mathcal{L} V(S, I, R) \leq c V(S, I, R)$ due to $V(S, I, R) \geq 4$ for $(S, I, R) \in \mathbb{D}$.

In what follows, to show that $P(\tau(\mathbb{D})=\infty)=1$, i.e., $P(\tau(\mathbb{D})<t)=0$. Now, introduce a new function $W \in C^{1,2}([s, \infty) \times \mathbb{D})$ by $W(t,(S, I, R))=\mathrm{e}^{-c(t-s)} V(S, I, R)$, where $c$ is defined as above. And hence

$$
\mathcal{L} W(t,(S, I, R))=\mathrm{e}^{-c(t-s)}[-c V(S, I, R)+\mathcal{L} V(S, I, R)] \leq 0,
$$

since $\mathcal{L} V(S, I, R) \leq c V(S, I, R)$. Denote $\tau_{n}:=\min \left\{\tau\left(\mathbb{D}_{n}\right), t\right\}$ and apply averaged Itô formula, we have

$$
\begin{aligned}
& E\left[\mathrm{e}^{c\left(t-\tau_{n}\right)} V\left(S\left(\tau_{n}\right), I\left(\tau_{n}\right), R\left(\tau_{n}\right)\right)\right] \\
& =E\left[\mathrm{e}^{c(t-s)} \mathrm{e}^{-c\left(\tau_{n}-s\right)} V\left(S\left(\tau_{n}\right), I\left(\tau_{n}\right), R\left(\tau_{n}\right)\right)\right] \\
& =E\left[\mathrm{e}^{c(t-s)} W\left[\tau_{n},\left(S\left(\tau_{n}\right), I\left(\tau_{n}\right), R\left(\tau_{n}\right)\right)\right]\right]
\end{aligned}
$$




$$
\begin{aligned}
= & \mathrm{e}^{c(t-s)} E\left[W\left[\tau_{n},\left(S\left(\tau_{n}\right), I\left(\tau_{n}\right), R\left(\tau_{n}\right)\right)\right]\right] \\
= & \mathrm{e}^{c(t-s)}[E W[s,(S(s), I(s), R(s))] \\
& \left.+E \int_{s}^{\tau_{n}} \mathcal{L} W(x,(S(x), I(x), R(x))) \mathrm{d} x\right] \\
\leq & \mathrm{e}^{c(t-s)} E W[s,(S(s), I(s), R(s))] \\
= & \mathrm{e}^{c(t-s)} E V[(S(s), I(s), R(s))] \\
\leq & \mathrm{e}^{c(t-s)} E V\left(S_{0}, I_{0}, R_{0}\right) .
\end{aligned}
$$

Using this fact and Equation (7), one can estimates

$$
\begin{aligned}
0 & \leq P(\tau(\mathbb{D})<t) \leq P\left(\tau\left(\mathbb{D}_{n}\right)<t\right)=P\left(\tau_{n}<t\right)=E\left(I_{\tau_{n}}<t\right) \\
& \leq E\left[\mathrm{e}^{c\left(t-\tau_{n}\right)} \frac{V\left(S\left(\tau\left(\mathbb{D}_{n}\right)\right), I\left(\tau\left(\mathbb{D}_{n}\right)\right), R\left(\tau\left(\mathbb{D}_{n}\right)\right)\right)}{\inf _{(S, I, R) \in \mathbb{D} \backslash \mathbb{W}_{n}} V(S, I, R) I_{\tau_{n}<t}}\right] \\
& \leq \mathrm{e}^{c\left(t-\tau_{n}\right)} \frac{E V\left(S_{0}, I_{0}, R_{0}\right)}{\inf _{(S, I, R) \in \mathbb{D} \mathbb{W}_{n}} V(S, I, R)} \\
& \leq \mathrm{e}^{c\left(t-\tau_{n}\right)} \frac{E V\left(S_{0}, I_{0}, R_{0}\right)}{2 n+2} \rightarrow 0 \text { as } n \rightarrow \infty,
\end{aligned}
$$

for all fixed $t \in[s,+\infty)$, because of the appearance of the function $I($.$) . Thus$ $P(\tau(\mathbb{D})<t)=P\left(\tau\left(\mathbb{D}_{n}\right)<t\right)=0$ for $\left(S_{0}, I_{0}, R_{0}\right) \in \mathbb{D}$ and $t \geq t_{0}$, that is, $P\left(\tau\left(\mathbb{D}_{n}\right)=\infty\right)=1$.

Then it proves the regularity and the global existence of the solution $(S(t), I(t), R(t)) \in \mathbb{D}$ and by means of Lemma 2.1 under the condition of (A), we also derive the uniqueness and continuity of the solution.

Similarly the above discussions, we only need to take the function $V(S, I, R)$ as $S-\ln S+(K-S)-\ln (K-S)$, and we can also obtain the same results under the condition of (B). Here, we omits the details.

This completes the proof of Theorem 3.1.

Remark 3.1. Because $I=S=R=0$ are undefined in the domain $\mathbb{D}$. In what follows, we distinguish three cases to investigate the solution of these special situations.

1) If $S=0$, then the system (5) will reduce to

$$
\left\{\begin{array}{l}
\mathrm{d} I(t)=(\mu K-(\alpha+\gamma+\mu) I(t)) \mathrm{d} t-\int_{0}^{h} f(s) I(t-s) \mathrm{d} s F(I(t), R(t)) \mathrm{d} W, \\
\mathrm{~d} R(t)=(\alpha I(t)-\mu R(t)) \mathrm{d} t+\int_{0}^{h} f(s) I(t-s) \mathrm{d} s F(I(t), R(t)) \mathrm{d} W,
\end{array}\right.
$$

with intial condition $\left(I_{0}, R_{0}\right) \in D_{1}=\{(I, R): I>0, R>0, I+R \leq K\}$. By using the similar analysis, we know that the above SDE is regular which implies there exists a unique global solution on $D_{1}$;

2) If $I=0$, then the system (5) will reduce to an ODE

$$
\left\{\begin{array}{l}
\mathrm{d} S(t)=\mu(K-S(t)) \mathrm{d} t \\
\mathrm{~d} R(t)=-\mu R(t) \mathrm{d} t
\end{array}\right.
$$


with intial condition $\left(S_{0}, R_{0}\right) \in D_{2}=\{(S, R): S>0, R>0, S+R \leq K\}$. By using the theory of ODE, we know that the above ODE is regular which implies there exists a unique global solution on $D_{2}$;

3) If $R=0$, then the system (5) will become

$$
\left\{\begin{aligned}
\mathrm{d} S(t)= & \left(-\beta S(t) \int_{0}^{h} f(s) I(t-s) \mathrm{d} s+\mu(K-S(t))\right) \mathrm{d} t \\
& -\int_{0}^{h} f(s) I(t-s) d s \times F(S(t), I(t)) \mathrm{d} W, \\
\mathrm{~d} I(t)= & \left(\beta S(t) \int_{0}^{h} f(s) I(t-s) \mathrm{d} s-(\alpha+\mu) I(t)\right) \mathrm{d} t \\
& +S(t) \int_{0}^{h} f(s) I(t-s) \mathrm{d} s \times F(S(t), I(t)) \mathrm{d} W,
\end{aligned}\right.
$$

with intial condition $\left(S_{0}, I_{0}\right) \in D_{3}=\{(S, I): S>0, I>0, S+I \leq K\}$. By using the similar analysis, we know that the above $S D E$ is regular which implies there exists a unique global solution on $D_{3}$.

\section{Global Stochastic Asymptotic Stability of Disease Free Equilibrium}

Theorem 4.1. Assume that $\int_{0}^{h} f(s) I(t-s) \mathrm{d} s \leq \frac{\alpha+\gamma+\mu}{K \beta} I(t)$ for all fixed $t \in[s, \infty)$, then the disease free equilibrium solution $\left(S_{1}, I_{1}, R_{1}\right)=(K, 0,0)$ of Equation (5) is globally stochastically stable on $\mathbb{D}$.

Proof. Notice that the assumption $\int_{0}^{h} f(s) I(t-s) \mathrm{d} s \leq \frac{\alpha+\gamma+\mu}{K \beta} I(t)$ for all fixed $t \in[s, \infty)$, and hence one can estimates that there exists a positive constant $C$ which satisfies $K \beta \int_{0}^{h} f(s) I(t-s) \mathrm{d} s \leq C \leq(\alpha+\gamma+\mu) I(t)$ for all fixed $t \in[s, \infty)$. Introduce a Lyapunov function

$$
V(S, I, R)=\frac{1}{2}(S+I+R-K)^{2}+C I .
$$

Just note that the infinitesimal generator $\mathcal{L}$ satisfies

$$
\begin{aligned}
\mathcal{L} V(S, I, R)= & \left.-\beta S \int_{0}^{h} f(s) I(t-s) \mathrm{d} s+\mu(K-S)\right)(S+I+R-K) \\
& +\left(\beta S \int_{0}^{h} f(s) I(t-s) \mathrm{d} s-(\alpha+\gamma+\mu) I\right)(S+I+R-K) \\
& +(\alpha I-\mu R)(S+I+R-K)+C\left(\beta S \int_{0}^{h} f(s) I(t-s) \mathrm{d} s-(\alpha+\gamma+\mu) I\right) \\
= & -\mu(K-S-I-R)^{2}+(K-S-I-R) \gamma I+C \beta S \int_{0}^{h} f(s) I(t-s) \mathrm{d} s \\
& -M I(\alpha+\gamma+\mu)-\gamma I^{2}-\gamma R I \\
= & -\mu(K-S-I-R)^{2}+\left(C \beta \int_{0}^{h} f(s) I(t-s) \mathrm{d} s-\gamma I\right) s \\
& +[K \gamma-C(\alpha+\gamma+\mu)] I-\gamma I^{2}-\gamma R I \\
\leq & -\mu(K-S-I-R)^{2}-\gamma I^{2}-\gamma R I,
\end{aligned}
$$

then $\mathcal{L} V(S, I, R)$ becomes negative definite on $\mathbb{D}$, and hence it completes the proof of Theorem 4.1 by applying Lemma 2.2. 
Remark 4.1. As we known, the basic reproduction number $R_{0}$ is one of the most important parameters in epidemiology, which reflects the expected number of secondary infections produced when one infected individual entered a fully susceptible population. If $R_{0}<1$ then the outbreak will disappear, on the other hand, if $R_{0}>1$ then the epidemic will spread a population. In this context, the basic reproduction number of the SIR model is $R_{0}=\frac{\beta K}{\alpha+\gamma+\mu}$.

\section{Stochastic Asymptotic Stability of Endemic Equilibrium}

If $R_{0}>1$ and $F_{i}\left(S_{2}, I_{2}, R_{2}\right)=0(i=1,2)$, then there exists a unique endemic equilibrium solution $\left(S_{2}, I_{2}, R_{2}\right)$ for the model (5), where

$$
\begin{aligned}
\left(S_{2}, I_{2}, R_{2}\right) & =\left(\frac{\alpha+\gamma+\mu}{\beta}, \frac{\mu}{\beta}\left(\frac{\beta K}{\alpha+\gamma+\mu}-1\right), \frac{\alpha}{\beta}\left(\frac{\beta K}{\alpha+\gamma+\mu}-1\right)\right) \\
& =\left(\frac{K}{R_{0}}, \frac{\mu}{\beta}\left(R_{0}-1\right), \frac{\alpha}{\beta}\left(R_{0}-1\right)\right) .
\end{aligned}
$$

Theorem 5.1. The endemic equilibrium solution, $\left(S_{2}, I_{2}, R_{2}\right)$ of the Equation (5) is stochastically asymptotically stable on $\mathbb{D}=\{(S, I, R): S>0, I>0, R>0, S+I+R \leq K\}$ under the assumption of $R_{0}>1$ for some $F_{i}(S, I, R)$ such that $F_{i}\left(S_{2}, I_{2}, R_{2}\right)=0$ and satisfies $G(S, I, R) \leq 0$, where

$$
\begin{aligned}
G(S, I, R)= & -\frac{\mu}{S+I+R}\left(S+I+R-S_{2}-I_{2}-R_{2}\right)^{2}-\frac{\gamma}{S+I+R}\left(I-I_{2}\right)^{2} \\
& -a \beta\left(S-S_{2}\right)^{2} \int_{0}^{h} f(s) I(t-s) \mathrm{d} s-b \mu\left(S-S_{2}\right)^{2}-b \mu\left(R-R_{2}\right)^{2} \\
& +\frac{a}{2} S^{2}\left(\int_{0}^{h} f(s) I(t-s) \mathrm{d} s\right)^{2} F_{1}^{2}(S, I, R) \\
& +\frac{b}{2}\left(\int_{0}^{h} f(s) I(t-s) \mathrm{d} s\right)^{2} F_{2}^{2}(S, I, R),
\end{aligned}
$$

and

$$
a=\left\{\begin{array}{l}
s>0, \quad \text { if }\left(S-S_{2}\right)\left(I-I_{2}\right)>0,\left(S-S_{2}\right)\left(\int_{0}^{h} f(s) I(t-s) \mathrm{d} s-I_{2}\right)>0 ; \\
0, \quad \text { if }\left(S-S_{2}\right)\left(I-I_{2}\right)<0,\left(S-S_{2}\right)\left(\int_{0}^{h} f(s) I(t-s) \mathrm{d} s-I_{2}\right)<0 ; \\
a>\frac{K^{2} \gamma}{\beta S_{2}}, \quad \text { if }\left(S-S_{2}\right)\left(I-I_{2}\right)<0,\left(S-S_{2}\right)\left(\int_{0}^{h} f(s) I(t-s) \mathrm{d} s-I_{2}\right)>0 ; \\
=0, \quad \text { if }\left(S-S_{2}\right)\left(I-I_{2}\right)>0,\left(S-S_{2}\right)\left(\int_{0}^{h} f(s) I(t-s) \mathrm{d} s-I_{2}\right)<0, \\
\qquad b=\left\{\begin{array}{l}
\inf _{(S, I, R) \in \mathbb{D}} \frac{\gamma}{\alpha(S+I+R)}, \quad \text { if }\left(S-S_{2}\right)\left(R-R_{2}\right)>0 ; \\
\frac{\gamma}{\alpha K}, \quad \text { if }\left(S-S_{2}\right)\left(R-R_{2}\right)<0 .
\end{array}\right.
\end{array}\right.
$$

Proof. It is a fact that the endemic equilibrium solution of system (5) exists if $R_{0}>1$ and $F_{i}\left(S_{2}, I_{2}, R_{2}\right)=0$. Introduce a Lyapunov function 


$$
\begin{aligned}
V(S, I, R)= & S+I+R-\left(S_{2}+I_{2}+R_{2}\right)-\left(S_{2}+I_{2}+R_{2}\right) \ln \frac{S+I+R}{S_{2}+I_{2}+R_{2}} \\
& +\frac{a}{2}\left(S-S_{2}\right)^{2}+\frac{b}{2}\left(R-R_{2}\right)^{2}+C
\end{aligned}
$$

on $\mathbb{D}$, where $a$ and $b$ are defined as Equations ((13) and (14)), $c$ is an arbitrary positive constant. An elementary computation leads to $V>0$ for any point $(S, I, R) \in \mathbb{D}$, and we have

$$
\begin{aligned}
\mathcal{L} V(S, I, R)= & \left.-\beta S \int_{0}^{h} f(s) I(t-s) \mathrm{d} s+\mu(K-S)\right]\left[1-\frac{S_{2}+I_{2}+R_{2}}{S+I+R}+a\left(S-S_{2}\right)\right] \\
& +\frac{a}{2} S^{2} \times\left[\int_{0}^{h} f(s) I(t-s) \mathrm{d} s\right]^{2} F_{1}^{2}(S, I, R)+\frac{b}{2}\left[\int_{0}^{h} f(s) I(t-s) \mathrm{d} s\right]^{2} F_{2}^{2} \\
& +\left[\beta S \int_{0}^{h} f(s) I(t-s) \mathrm{d} s-(\alpha+\gamma+\mu) I\right]\left[1-\frac{S_{2}+I_{2}+R_{2}}{S+I+R}\right] \\
& +(\alpha I-\mu R)\left[1-\frac{S_{2}+I_{2}+R_{2}}{S+I+R}+b\left(R-R_{2}\right)\right] .
\end{aligned}
$$

From the following formulas and the definitions of $a, b$ can help to simplify $\mathcal{L}(S, I, R)$

i) $\mu(K-I-S-R)-\gamma I=-\mu\left(S+I+R-S_{2}-I_{2}-R_{2}\right)-\gamma\left(I-I_{2}\right)$;

ii)

$$
\begin{aligned}
& -\beta S \int_{0}^{h} f(s) I(t-s) \mathrm{d} s+\mu(K-S) \\
& =-\beta\left(S-S_{2}\right) \int_{0}^{h} f(s) I(t-s) \mathrm{d} s-\beta S_{2}\left(\int_{0}^{h} f(s) I(t-s) \mathrm{d} s-I_{2}\right)-\mu\left(S-S_{2}\right)
\end{aligned}
$$

iii) $\alpha+\beta+\mu=\beta S_{2}$;

iv) $\alpha I-\mu R=\alpha\left(I-I_{2}\right)-\mu\left(R-R_{2}\right)$.

Then

$$
\begin{aligned}
\mathcal{L} V= & {[\mu(K-S-I-R)-\gamma I]\left[1-\frac{S_{2}+I_{2}+R_{2}}{S+I+R}\right]+a\left(S-S_{2}\right)\left[-\beta\left(S-S_{2}\right)\right.} \\
& \left.\times \int_{0}^{h} f(s) I(t-s) \mathrm{d} s-\beta S_{2}\left(\int_{0}^{h} f(s) I(t-s) \mathrm{d} s-I_{2}\right)-\mu\left(S-S_{2}\right)\right] \\
& +\frac{a}{2} S^{2}\left[\int_{0}^{h} f(s) I(t-s) \mathrm{d} s\right]^{2} F_{1}^{2}(S, I, R)+\frac{b}{2}\left[\int_{0}^{h} f(s) I(t-s) \mathrm{d} s\right]^{2} F_{2}^{2} \\
= & -\frac{\mu}{S+I+R}\left[S+I+R-S_{2}-I_{2}-R_{2}\right]^{2}-\frac{\gamma}{S+I+R}\left(I-I_{2}\right)^{2} \\
& -\frac{\gamma}{S+I+R}\left(S-S_{2}\right)\left(I-I_{2}\right)-\frac{\gamma}{S+I+R}\left(I-I_{2}\right)\left(R-R_{2}\right) \\
& -a \beta\left(S-S_{2}\right)^{2} \int_{0}^{h} f(s) I(t-s) \mathrm{d} s-a \beta S_{2}\left(S-S_{2}\right)\left(\int_{0}^{h} f(s) I(t-s) \mathrm{d} s-I_{2}\right) \\
& +\frac{a}{2} S^{2}\left[\int_{0}^{h} f(s) I(t-s) \mathrm{d} s\right]^{2} F_{1}^{2}(S, I, R)+\frac{b}{2}\left[\int_{0}^{h} f(s) I(t-s) \mathrm{d} s\right]^{2} F_{2}^{2} \\
\leq & -\frac{\mu}{S+I+R}\left[S+I+R-S_{2}-I_{2}-R_{2}\right]^{2}-\frac{\gamma}{S+I+R}\left(I-I_{2}\right)^{2} \\
& -a \mu\left(S-S_{2}\right)^{2}+b\left(R-R_{2}\right)\left[\alpha\left(I-I_{2}\right)-\mu\left(R-R_{2}\right)\right] \\
& +b \alpha\left(I-I_{2}\right)\left(R-R_{2}\right)-b \mu\left(R-R_{2}\right)^{2}-a \mu\left(S-S_{2}\right)^{2} \\
& -a \beta\left(S-S_{2}\right)^{2} \int_{0}^{h} f(s) I(t-s) \mathrm{d} s-b \mu\left(R-R_{2}\right)^{2} \\
+ & \frac{a}{2} S^{2}\left[\int_{0}^{h} f(s) I(t-s) \mathrm{d} s\right]^{2} \times F_{1}^{2}(S, I, R)+\frac{b}{2}\left[\int_{0}^{h} f(s) I(t-s) \mathrm{d} s\right]^{2} F_{2}^{2} .
\end{aligned}
$$


$\mathcal{L} V(S, I, R)=0$ if and only if $(S, I, R)=\left(S_{2}, I_{2}, R_{2}\right)$ and by the given condition one can obtain $\mathcal{L} V<0$ on $\mathbb{D} \backslash\left(S_{2}, I_{2}, R_{2}\right)$. Therefore $\mathcal{L} V(S, I, R)$ is negative definite on $\mathbb{D}$ for some suitable $F_{i}(S, I, R)$. Then Lemma 2.2 (ii) leads to the stochastically asymptotical stability of the endemic equilibrium with $R_{0}>1$ and for some suitable functions $F_{i}(S, I, R)$ such that $F_{i}(S, I, R)$ satisfies Equation (12) and $F_{i}\left(S_{2}, I_{2}, R_{2}\right)=0$

\section{Example}

In this section, we visualize our results with some simulation to confirm them. Due to the difficulty of the research on the drawing of the disease equilibrium point, many scholars have not given the relevant examples. Along this clue, we only give the figures of the disease-free equilibrium point (Figure 1). We consider the special case $\int_{0}^{1} f(s) I(t-s) \mathrm{d} s=I(t)$ which only satisfies the condition of Theorem $4.1 \int_{0}^{h} f(s) I(t-s) \mathrm{d} s \leq \frac{\alpha+\gamma+\mu}{K \beta} I(t)$, that is, $\frac{K \beta}{\alpha+\gamma+\mu}<1$, and hence we can obtain the disease free equilibrium solution $\left(S_{1}, I_{1}, R_{1}\right)=(K, 0,0)$ of Equation (5) is globally stochastically stable on $\mathbb{D}$. In the simulation, the parameters are chosen as follows

$$
K=1000, \quad \mu=1 / 75=0.013, \quad \alpha=52, \quad \gamma=52, \quad \beta=0.05 .
$$

\section{Acknowledgements}

Special thanks to the anonymous referees for very useful suggestions. The research has been supported by the Natural Science Foundation of China
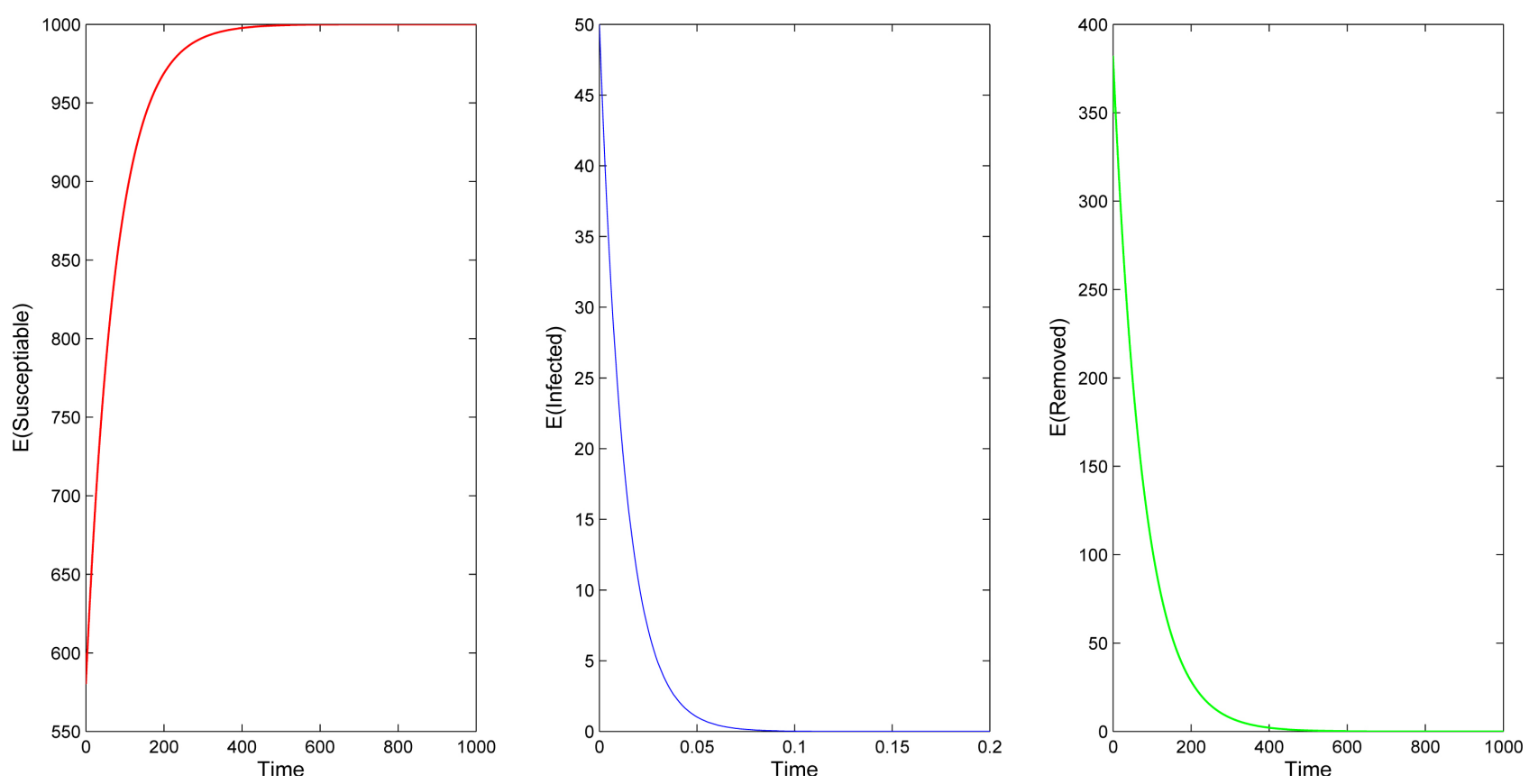

Figure 1. The disease free equilibrium $(S, I, R)=(1000,0,0)$ is globally stochastically asymptotically stable since $\frac{K \beta}{\alpha+\gamma+\mu}<\frac{K \beta}{\gamma}=0.97<1$ for $\alpha=52, \mu=0.013, K=1000, \beta=0.05$ and $\gamma=52$. 
(11361004). Xian-Hua Xie is supported by the Bidding Project of Gannan Normal University (16zb01). all of the authors are supported by the Key Laboratory of Jiangxi Province for Numerical Simulation and Emulation Techniques.

\section{Conflict of Interests}

The authors declare that the study was realized in collaboration with the same responsibility.

\section{Competing Interests}

The authors declare that they have no competing interests regarding the publication of this paper.

\section{Authors Contributions}

All of the authors, XHX, LM and JFX contributed substantially to this paper, participated in drafting and checking the manuscript, and have approved the version to be published.

\section{References}

[1] Allen, E.J., Allen, L.J.S. and Schurz, H. (2005) A Comparison of Persistence-Time Estimation for Discrete and Continuous Stochastic Population Models That Include Demographic and Environmental Variability. Mathematical Biosciences, 196, 1438. https://doi.org/10.1016/j.mbs.2005.03.010

[2] Allen, E.J. (1999) Stochastic Differential Equations and Persistence Time for Two Interacting Populations. Dynamics of Continuous, Discrete and Impulsive Systems, 5, 271-281.

[3] Arciniega, A. and Allen, E. (2004) Shooting Methods for Numerical Solution of Stochastic Boundary-Value Problems. Stochastic Analysis and Applications, 22, 1295-1314. https://doi.org/10.1081/SAP-200026465

[4] Arnold, L. (1974) Stochastic Differential Equation. Wiley, New York.

[5] Bartholomew, D.J. (1982) Stochastic Models for Social Processes. Wiley, New York.

[6] Bartlett, M.S., Gower, J.C. and Leslie, P.H. (1960) A Comparison of Theoretical and Empirical Results for Some Stochastic Population Models. Biometrika, 47, 1-11 https://doi.org/10.1093/biomet/47.1-2.1

[7] Beretta, E., Kolmanovskii, V. and Shaikhet, L. (1998) On the General Structure of Epidemic System, Global Asymptotic Stability. Computers \& Mathematics with Applications, 12, 677-694. https://doi.org/10.1016/0898-1221(86)90054-4

[8] Beretta, E. and Takeuchi, Y. (1995) Global Stability of an SIR Epidemic Model with Time Delays. Journal of Mathematical Biology, 33, 250-260 https://doi.org/10.1007/BF00169563

[9] Carletti, M. (2002) On the Stability Properties of a Stochastic Model for PhageBacteria Interaction in Open Marine Environment. Mathematical Biosciences, 175, 117-131. https://doi.org/10.1016/S0025-5564(01)00089-X

[10] Gard, T.C. (1988) Introduce to Stochastic Differential Equations. Marcel Dekker, Basel.

[11] Golec, J. and Sathananthan, S. (2003) Stabilitty Analysis of a Stochastic Logistic Model. Mathematical and Computer Modelling, 38, 585-593 
https://doi.org/10.1016/S0895-7177(03)90029-X

[12] Hanson, F.B. and Tuckwell, H.C. (1981) Logistic Growth with Random Density Independent Disasters. Theoretical Population Biology, 19, 1-11.

https://doi.org/10.1016/0040-5809(81)90032-0

[13] Hethcote, H.W. (1976) Qualitative Analyses of Communicable Disease Models. Mathematical Biosciences, 28, 335-356. https://doi.org/10.1016/0025-5564(76)90132-2

[14] Schurz, H. (2001) Moment Attractivity, Stability and Contractivity Exponents of Stochastic Dynamical Systems. Discrete and Continuous Dynamical SystemsSeries A, 7, 487-515. https://doi.org/10.3934/dcds.2001.7.487

[15] Schurz, H. (2007) Modelling Analysis and Discretization of Stochastic Logistic Equations. International Journal of Numerical Analysis and Modeling, 4, 180-199.

[16] Tornatore, E., Buccellato, S.M. and Vetro, P. (2005) Stability of a Stochastic SIR System. Physica A: Statistical Mechanics and its Applications, 354, 111-126. https://doi.org/10.1016/j.physa.2005.02.057

[17] Zhang F., Gao, S., Liu, Y., et al. (2016) Dynamics of a Nonautonomous SIR Model with Time-Varying Impulsive Release and General Nonlinear Incidence Rate in a Polluted Environment. Applied Mathematics, 7, 681-693. https://doi.org/10.4236/am.2016.77062

[18] Zhang, Y., Chen, S., Gao, S., et al. (2017) A New Non-Autonomous Model for Migratory Birds with Leslie-Gower Holling-Type II Schemes and Saturation Recovery rate. Mathematics and Computers in Simulation, 132, 289-306.

https://doi.org/10.1016/j.matcom.2016.07.015

[19] Zhao, Y. and Yuan, S.L. (2017) Optimal Harvesting Policy of a Stochastic Two-Species Competitive Model with Lévy Noise in a Polluted Environment. Physica A: Statistical Mechanics and its Applications, 477, 20-33.

https://doi.org/10.1016/j.physa.2017.02.019

\section{Scientific Research Publishing}

Submit or recommend next manuscript to SCIRP and we will provide best service for you:

Accepting pre-submission inquiries through Email, Facebook, LinkedIn, Twitter, etc. A wide selection of journals (inclusive of 9 subjects, more than 200 journals)

Providing 24-hour high-quality service

User-friendly online submission system

Fair and swift peer-review system

Efficient typesetting and proofreading procedure

Display of the result of downloads and visits, as well as the number of cited articles

Maximum dissemination of your research work

Submit your manuscript at: http://papersubmission.scirp.org/

Or contact am@scirp.org 